\title{
Erratum: Sánchez-Sánchez, B., et al. The Female Sexual Function Index: Transculturally Adaptation and Psychometric Validation in Spanish Women. Int. J. Environ. Res. Public Health 2020, 17(3), 994
}

\author{
Beatriz Sánchez-Sánchez ${ }^{\mathbb{D}}$, Beatriz Navarro-Brazález ${ }^{\mathbb{D}}$, Beatriz Arranz-Martín, \\ Óscar Sánchez-Méndez $\mathbb{D}^{\mathbb{D}}$, Irene de la Rosa-Díaz * and María Torres-Lacomba $\mathbb{D}$ \\ Physiotherapy in Women's Health (FPSM) Research Group, Physiotherapy Department, Faculty of Medicine \\ and Health Sciences, University of Alcalá, Alcalá de Henares, 28805 Madrid, Spain; \\ beatriz.sanchez@uah.es (B.S.-S.); b.navarro@uah.es (B.N.-B.); beatriz.arranz@edu.uah.es (B.A.-M.); \\ osmfisio@gmail.com (Ó.S.-M.); maria.torres@uah.es (M.T.-L.) \\ * Correspondence: fisioterapia.mujer@uah.es
}

Received: 13 May 2020; Accepted: 15 June 2020; Published: 17 June 2020

The authors wish to make the following correction to their paper published in the International Journal of Environmental Research and Public Health [1]:

\section{Change in Main Body Paragraphs}

There are two mistakes in this article [1]:

On page 2, in the first paragraph of: 2.1. Translational and Cultural Adaptation subsection (2. Materials and Methods section), the sentence "This study (OE21/2013) was approved by «BLINDED» Hospital's Clinical Research Ethics Committee in «BLINDED».".

Will be corrected to: "This study (OE21/2013) was approved by Príncipe de Asturias Hospital's Clinical Research Ethics Committee in Alcalá de Henares (Madrid).".

On page 3, in the first paragraph of: 2.2. Participants and Procedure subsection (2. Materials and Methods section), the sentence "All women who were assessed by the «BLINDED» Research Group of the «BLINDED» and ... ".

Will be corrected to: "All women who were assessed by Physiotherapy in Women's Health Research Group of Alcalá University (Madrid, Spain) and ... ".

\section{References}

There is one mistake:

On page 13, Reference number 33: “Sánchez, B.S.; Lacomba, M.T.; Brazález, B.N.; Téllez, E.C.; Da Costa, S.P.; Ortega, C.G. Responsiveness of the Spanish Pelvic Floor Distress Inventory and Pelvic Floor Impact Questionnaires Short Forms (PFDI-20 and PFIQ-7) in women with pelvic floor disorders. Eur. J. Obstet. Gynecol. Reprod. Biol. 2015, 190, 20-25.".

Will be corrected to: "Sánchez-Sánchez, B.; Torres-Lacomba, M.; Navarro-Brazález, B.; Cerezo-Téllez, E.; Pacheco-Da-Costa, S.; Gutiérrez-Ortega, C. Responsiveness of the Spanish Pelvic Floor Distress Inventory and Pelvic Floor Impact Questionnaires Short Forms (PFDI-20 and PFIQ-7) in women with pelvic floor disorders. Eur. J. Obstet. Gynecol. Reprod. Biol. 2015, 190, 20-25.".

The authors would like to apologize for any inconvenience caused to the readers by these changes.

Conflicts of Interest: The authors declare no conflict of interest. 


\section{Reference}

1. Sánchez-Sánchez, B.; Navarro-Brazález, B.; Arranz-Martín, B.; Sánchez-Méndez, Ó.; de la Rosa-Díaz, I.; Torres-Lacomba, M. The female sexual function index: transculturally adaptation and psychometric validation in Spanish women. Int. J. Environ. Res. Public Health 2020, 17, 994. [CrossRef] [PubMed] 